\title{
An Integrated Approach to Support Knowledge Representation, Sharing and Perception over Web2.0
}

\author{
lyad AlAgha \\ The Islamic University of Gaza \\ Gaza Strip, Palestine
}

\begin{abstract}
Web 2.0 technologies provide the communication medium which allows collaborators to share and communicate ideas. These technologies, however, exhibit limited support for knowledge transfer which is more than a communication problem. It is still unclear how people can communicate, over the Web 2.0, cognitive activities which they undertake during the knowledge acquisition process.

In this work, we present an integrated approach for knowledge sharing that addresses the needs of both knowledge providers and recipients. It combines both concept mapping and dynamic annotation to enable for effective construction and perception of knowledge. The approach begins by helping knowledge providers visualize their own experience using a tool we named "MindGate". Afterwards, an Ontology-based model is used to convert the visual representation of knowledge to a machine-readable format. This format can then be published and reused by Internet users through a prototype social network called "SocialMinds". We have tested our approach with three research students who used our approach to carry out research tasks. The analysis of user behavior, activity and tool usage proved the potential of our approach to facilitate knowledge sharing and support both individual and group work.
\end{abstract}

\section{General Terms}

Knowledge Representation, Semantic Web, Human-Computer Interaction, E-learning, Collaborative Learning

\section{Keywords}

Knowledge representation, Semantic Web, Ontology, Collaborative Learning

\section{INTRODUCTION}

Knowledge sharing is the activity through which knowledge (i.e., information, skills, or expertise) is exchanged among people. With the popular use of computers in recent years, technologies such as knowledge bases, knowledge repositories, group decision support systems, intranets, and computer-supported cooperative work have been introduced to further enhance such efforts.

One of the main challenges of knowledge sharing is how knowledge can be transferred from one entity to another [1]. Knowledge transfer aims to organize, create, capture or distribute knowledge and ensure its availability for future users [2]. However, knowledge transfer can be quite complex because it seeks to articulate knowledge which resides in human mind and represent it in a format that can be communicated with others [3]. Knowledge consists of not only information but also the cognitive associations and the reasoning made while processing information [4]. While information can be easily transferred, cognitive activities are considered difficult to share and communicate.
With the advent of Web 2.0, the concept of knowledge sharing has evolved towards a vision more based on people participation and social networking. Through social networking sites, people can share different types of information such as hyperlinks, documents, quotes and personal comments. Web 2.0 technologies provide the communication medium which allows collaborators to share and communicate ideas [5]. These technologies, however, exhibit limited support for knowledge transfer which is more than a communication problem. It is still unclear how people can communicate, over Web 2.0 technologies, cognitive activities which they undertake during the knowledge acquisition process $[6,7]$.

We believe that sharing of cognitive activities over Web 2.0 can offer valuable benefits: First it enables collaborators to gain deep understanding of knowledge by accessing and tracing the associations and analytics made by the knowledge creators. Second, by sharing their cognitive activities, people may gain feedback from peers on potential defects, misconceptions or conflicts in their arguments or cognitive structures. Furthermore, sharing the structure of knowledge and human cognition in a machine understandable format can provide opportunities to build intelligent web applications. For example, a recommender service can utilize the structure of individual's knowledge in order to match users who have similar or related interests. Another web application may offer automatic validation of someone's knowledge by comparing it against the structure of an expert's knowledge.

The above discussion triggers a challenge for Web 2.0 technologists, which is how to support the sharing of cognitive processes that users perform while building knowledge. While it is possible to share the knowledge outcomes in different formats (e.g. as a note, a blog entry, a document or a sketch), it is difficult to share the implicit cognitive analytics which led to these outcomes.

In an endeavor to support knowledge sharing over Web 2.0, we propose an approach that utilizes visual modeling and ontologies to aid users in representing and structuring their knowledge in a format that can be shared on Web 2.0. It also provides the appropriate mechanisms to enable knowledge recipients to access and process shared knowledge easily with minimal effort. Through a set of tools presented in this paper, we will show how our approach works in practice to allow for seamless transfer of knowledge from providers to recipients.

\section{BACKGROUND AND RELATED WORK}

Knowledge sharing has been identified as a major focus area for knowledge management. Information systems such as intranets, distributed libraries or groupware applications are all proposed to support knowledge sharing. Reports, however, show that the use of these systems does not result in significant improvement in knowledge sharing [5, 8]. While 
these systems focus merely on boosting information distribution and communication, knowledge sharing involves other activities which remain largely unexplored.

Knowledge sharing presumes a relationship between two parties: the one that possess knowledge and is willing to share it, and the other who will receive and perceive the shared knowledge. The first party needs to represent knowledge in a format that can be shared and perceived easily by the other party [9]. However, Knowledge is not a raw data that can be passed around freely. It is an act of reconstruction and reasoning which cannot be easily represented in a format that can be posted on the Web [10]. Knowledge representation has been always identified as a barrier to knowledge sharing and communication. Bukowitz et al. [11] identified the stages that need to be met to achieve successful sharing of knowledge as the following:

- Articulation: the knowledge provider can describe the information.

- Awareness: the recipient must be aware that knowledge is available.

- Access: the knowledge recipient can access knowledge easily.

These stages address the needs of both the knowledge provider, who is responsible for articulating knowledge successfully, as well as the knowledge recipient, who should be able to access and interpret knowledge easily. If we are to apply these steps for knowledge sharing on Web2.0, it is necessary to support the appropriate tools and methods to enable for easy representation, sharing, access and interpretation of knowledge. While these stages have been explored individually in previous research efforts, it is hard to find a single integrated work that addresses this whole process as the one proposed in this work.

\subsection{Knowledge Representation}

As discussed earlier, knowledge representation remains the biggest challenge for effective knowledge sharing. The inability to articulate knowledge clearly will cause the whole process to fail as the recipients will be unable to perceive knowledge as desired. Existing research has introduced a considerable number of knowledge representation techniques such as frames, rules, tagging [12] and more recently Semantic Web based languages (e.g. RDF, OWL) [13]. In what follows, we discuss two popular tools that facilitate knowledge representation and that inspired the work presented in this paper. We also discuss the feasibility of these tools for knowledge sharing on Web 2.0.

\subsubsection{Graphical Knowledge Representation}

Graphical knowledge representation has been widely used to represent relationships between concepts, ideas, actions, decisions or even any information artefacts such as images and files. Examples of visual techniques of knowledge representation include concept maps, mind maps and spider diagrams [14]. One of the main characteristics of concept mapping is that it allows representing the associations between things in a hierarchal fashion so that most general concepts come at the top while more specific, less general concepts are arranged hierarchically below. Connector lines between concepts can be named with keywords or phrases that summarize the relationship between the topics they connect [15].

Mind maps, on the other hand, are used to slice and dice the central concept in a large number of different ways, or explore branches out from the centre [16]. Mind maps are generally more suitable for personal note taking and reviewing $[14,15$, 17]. Other techniques of knowledge representation may include conceptual diagrams [18] and visual metaphors [19], although they are less common when compared with concept and mind mapping [17].

Visual techniques of knowledge representation exhibit enormous benefits for learning. Previous studies have shown that they can effectively foster revision and reconstruction of knowledge [20] as well as encourage creativity and selfexpression [21, 22]. Some efforts have tried to adapt concept mapping for collaborative learning. For example, CmapTools [23] is a software package that allow internet users to create concept maps and publish them as web pages. Compendium [24] is a collection of tools that enable groups of users to collectively elicit and organize knowledge using concept mapping. Hwang et al. [25] presented a tool that enables learners to collaboratively develop concept maps through mobile devices. ClaiMaker [26] is a web-based system for individuals or distributed communities to publish and contest ideas and arguments.

All the previous efforts sought to facilitate knowledge representation and sharing by means of visual modeling and concept mapping. However, they do not address the needs of the knowledge recipients who may encounter difficulties to perceive knowledge from its visual representation. Eppler [17] indicated that concept mapping is generally useful for personal use only to support information revision and recall. Because they probe an individual's organization of knowledge, concept maps may not be easily understandable by others who know nothing about the context and the circumstances which informed the structure of knowledge. In addition, it may be difficult to trace concept maps when they become large and dense. Thus, it is not enough to share the "big picture" of knowledge, as depicted by concept or mind maps, to support collaborative learning. We argue that it is necessary to give users access to the context, circumstances and analysis which drove an individual to structure knowledge in a particular manner. However, this premise impose extra challenges such as how all these details can be published and accessed over the internet without causing knowledge recipients to be overwhelmed or distracted.

\subsubsection{Annotation Systems}

Annotation systems enable users to attach metadata (e.g. comment, explanation, presentational mark-ups) to text, images or other data. Annotations provide an opportunity to associate the individual perceptions and views to original data [27]. To support shared understanding of user-annotations across Web applications and to avoid duplication and reinvention wherever possible, several models have been proposed to describe metadata annotations, such as Dublin Core [28] and ontologies.

Collaborators who access shared annotations can view original data or document content along with the user-added annotations. Previous research $[29,30]$ suggested that sharing user annotations with collaborators can facilitate collaborative learning by making individual knowledge to public ones. Thus, knowledge accumulated its value when it is shared. Recently, several annotation systems have been proposed to enhance knowledge sharing on Web 2.0 (e.g. [31-33]). While these systems can be powerful tools for collaborative learning, the scope of knowledge sharing is limited only to the content of the document being annotated. While reading a document, it is hard for the user to recall all the annotations attached to different documents. In addition, annotations that are attached 
at fine granular levels (e.g. paragraph, sentence) do not explicitly reveal the broad structure of knowledge and how different documents have been cognitively linked to make the user's knowledge.

Previous research addressed this limitation by introducing formal and ontological annotations [34] which have formally defined constraints and predefined terms. With formal annotations, it is possible to assist the user in revisiting the structure of knowledge by exploiting the predefined relationships between annotations. For example, an intelligent Web application can link different documents by inferring about the relationships between the attached annotations. In the last years, ontology based document annotation has been widely investigated to support intelligent information retrieval (e.g. [35], [36]) and extraction techniques (e.g. [37], [38]). Our approach has a different design goal, which is how to enable users on the Web to publish, share and reuse the cognitive activities (e.g. conclusions, comments, misconceptions, analysis, and associations). Thus, this work uses ontologies to annotate, not the documents, but the knowledge graph constructed by the users while reading documents.

In light of the above discussion, there are still research questions that need to be considered when it comes to webbased knowledge representation and sharing:

- How can people visually represent knowledge in a format that can be easily perceived by other users on the Web?

- How can knowledge representation be published and shared over social networking sites?

- How can collaborators access and perceive shared knowledge easily and without being cognitively overloaded?

In this paper, we propose an approach that combines both concept mapping and dynamic annotation to enable for effective construction and perception of knowledge. The approach begins by facilitating knowledge providers to visualize their own experience using a concept mapping tool. An Ontology-based model is then used to convert the visual representation of knowledge into a standardized and a machine-readable format. To enable users to access and interpret knowledge constructed by others, we designed a special approach that converts the published knowledge graph into annotations. These annotations will be embedded inside the documents which knowledge was originally gained from. The intention is to enable knowledge recipients to access and navigate the source documents in a way that reflects the analysis and the reasoning made by knowledge providers.

Following the criteria proposed by Bukowitz et al. [11] for successful sharing of knowledge, our solution to support knowledge transfer over Web 2.0 consists of three consecutive stages each of which addresses one of the three steps: articulation, awareness and access. The following subsections discuss these stages in detail.

\section{KNOWLEDGE ELICITATION AND ARTICULATION}

The first stage of our approach aims to assist knowledge providers in representing their knowledge in a format that can be published and shared over the Internet. To achieve this purpose, it is necessary first to understand how users process information and construct knowledge while learning. A typical user aiming to learn about some topic may refer to several resources such as a Web page giving an overview on the topic, an online course offering structured learning approach, and a research paper discussing the topic from a research perspective. Meanwhile, the user progressively builds knowledge by making links between these resources. For example, he may link a definition from one webpage with the explanation found in the online course. He may also find that some resources elaborate, and thus complement, the topic introduced by other resources.

Obviously, the overall knowledge map that exists in the user's mind is composed of the inter-related pieces of information as well as the conceptual links made between them. This knowledge map represents the user's own experience, which is likely to be different from a user to another due to their individual differences and the different learning paths they follow. It is obvious that the knowledge map, generated from the above scenario, always resides in the user mind. This implies that the user needs to retain all details and relationships in mind and recall them whenever knowledge needs to be revised or reviewed.

To allow the user to represent the structure of knowledge, we built a learning tool called "MindGate". MindGate is a PDFfile reader with additional capabilities to support knowledge representation and sharing. While reading documents, MindGate enable users to structure the way they process and link information content. The main window of MindGate is shown in Figure 1: PDF documents can be opened and displayed on the middle area (Figure 1.A). The panel on the left is a concept mapping area that enables the user to structure knowledge and link the documents being read. The structure of knowledge can be viewed as a map or graph with the documents providing vertices and the relations providing edges (Figure 1.C).

MindGate facilitates knowledge elicitation and articulation as the following: when the user finds any information of interest, he/she can make a reference to that information by creating a highlighting box over it (Figure 1.B), dragging and dropping it over the drawing area. This will create node, or a vertex, denoted by a thumbnail of the containing document. Each node also works as a bookmark that when clicked, the original document opens and scrolls to the location where the referenced segment resides. This enables the user to rapidly access information of interest by clicking on the corresponding nodes on the knowledge map.

MindGate also allows the user to attach personal notes to each node. This can be done through an input form that pops up when right-clicking any graph node. In addition, the panel on the bottom left corner (Figure 1.E) shows a miniature view of the concept mapping area. It allows the user to navigate through or zoom in/out the map quickly, especially when the map is large or complicated. As the learning process goes on, the user creates more nodes and links them. Links can also be named to reflect the types of relationships so that the user does not have to retain them in mind. The names of links indicate the relationships that hold between parts of documents from the user's perspective. For example, a user may decide that one document extends the topic discussed in another document, and hence represents this relation as an edge linking the two nodes. Eventually, the graph built on the mind-mapping area will model the user's workflow and the transitions between documents. In fact, the constructed map mimics the user's cognitive structure by showing how he/she managed to organize and link different resources of information. 


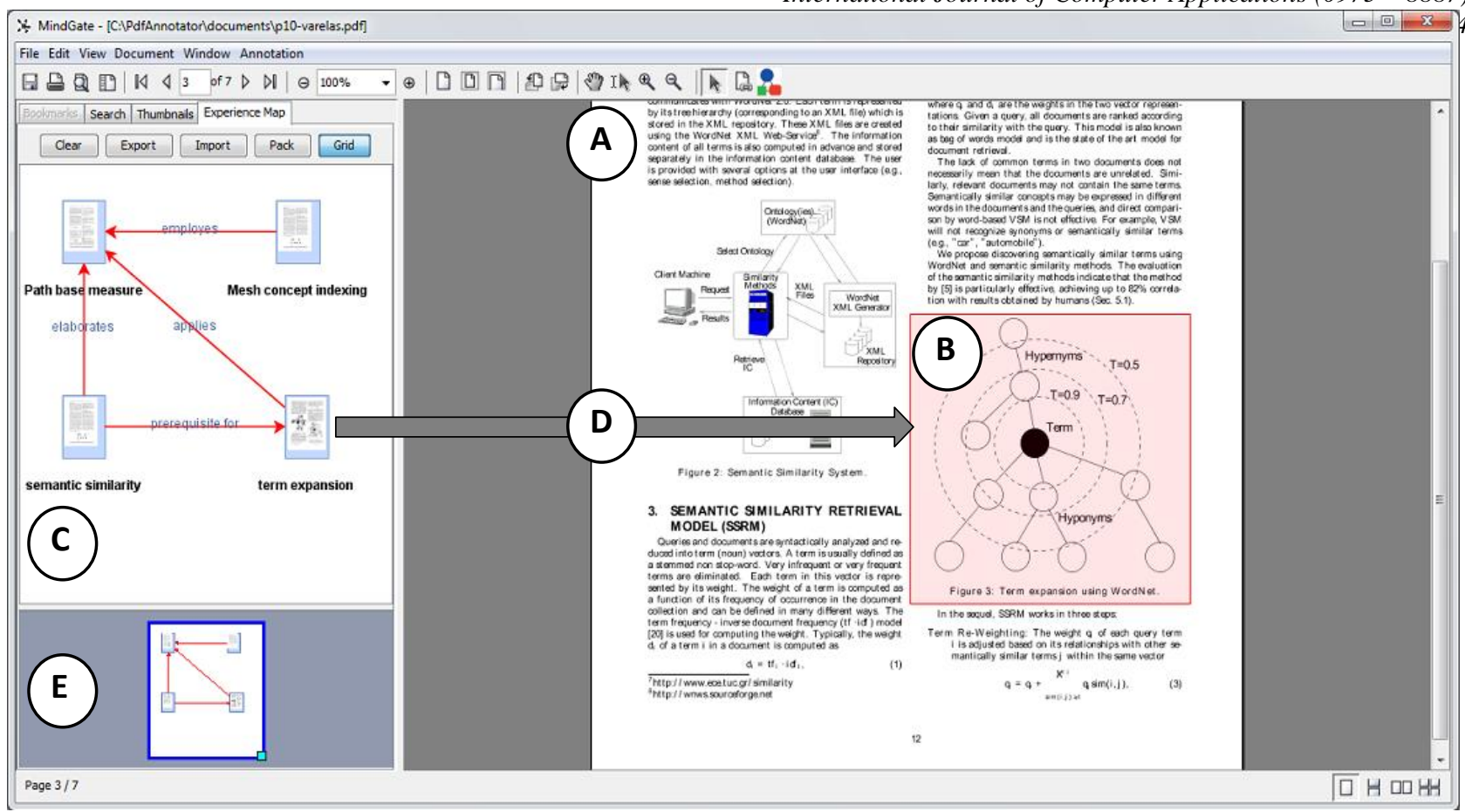

Fig 1: MindGate tool: A) PDF document view. B) A part of the document highlighted by the user. C) A knowledge map whereas documents are represented as nodes and relationships are represented as links. D) Each node is linked to its source information. E) A miniature view allowing for rapid zooming and transition within the map

\section{ONTOLOGY BASED REPRESENTATION OF KNOWLEDGE}

The concept-mapping area of the MindGate tool enables the user to plan the learning path in a way that shows not only the resources that he/she referred to, but also the cognitive links made while processing these resources.

The next stage is to represent the constructed graph in a transferable format which can be easily published and accessed over the Web. For that purpose, we propose an ontology based model to convert the graph into an RDF format. The model offers twofold benefits: First, it serves as a reference initiative that helps in structuring and standardizing the constructed maps. Second, if user maps are annotated by means of terms from common domain ontologies, ontological relationships can be exploited in the provision of intelligent services. Our ontology based model consists of three ontologies:

1) Domain ontology: While building the knowledge map, the user can annotate graph nodes with domain terms that best describe the topic(s) of the referenced resource. To help standardize these annotations, a domain ontology, which defines the concepts enclosed in a particular domain of knowledge, can be used. Domain terms are represented as ontology classes, and those are structured hieratically so that parent classes denote more general topics, while child classes demote sub or more specific topics.

Annotation of document content using domain ontologies can support topic-based categorization of map contents, semanticbased search and retrieval. For an evaluation purpose, we configured the MindGate tool to use a domain ontology that models the ACM classification of computer science topics. The annotation process is done easily through the user interface by providing a tree structure of the domain ontology and allowing the user to choose terms from (see Figure 2). We emphasize here that the tool can be reconfigured to support different domain ontologies.
2) Information Content Ontology: this defines the metadata used to describe the components of the user map. For example, every node has metadata describing its information content such as the source document that it refers to, the attached names, tags and user notes, incoming and outgoing edges with other nodes. Metadata defined in this ontology is used to store and reconstruct the user map.

3) Graphical attributes Ontology: when the RDF representation of a user map is publically shared, other users should be able to import and reconstructed the map on their machines exactly as it was constructed on the owner's machine. In order to maintain and standardize the visual details of the map, the graphical attributes ontology offers metadata describing information about the various styles, colors, positions and sizes of both nodes and edges. Note that the metadata defined in graphical attributes ontology could be integrated into the information content ontology. However, the intention to keep it separate was to keep the graphical details separate from the data model. This allows for altering the graphical representation without making any changes to the metadata describing data model. At any point, the user can save the constructed map and retrieve it at a later time. The work is saved as an RDF file whereas all referenced information, graphical details, relationships and annotations are represented as RDF triples using metadata from the three Ontologies. Importing the RDF file to the system will cause the graph map to be reconstructed.

\section{ADAPTATION OF CONTENT}

As mentioned earlier, mere concept mapping has the limitation of being sometimes hard to comprehend by knowledge recipients. This is because it only shows an abstract view of the knowledge structure without exposing the context from which knowledge was gained. Only the creator of the concept map will be able to tell exactly how and why the map was organized in a particular manner. 


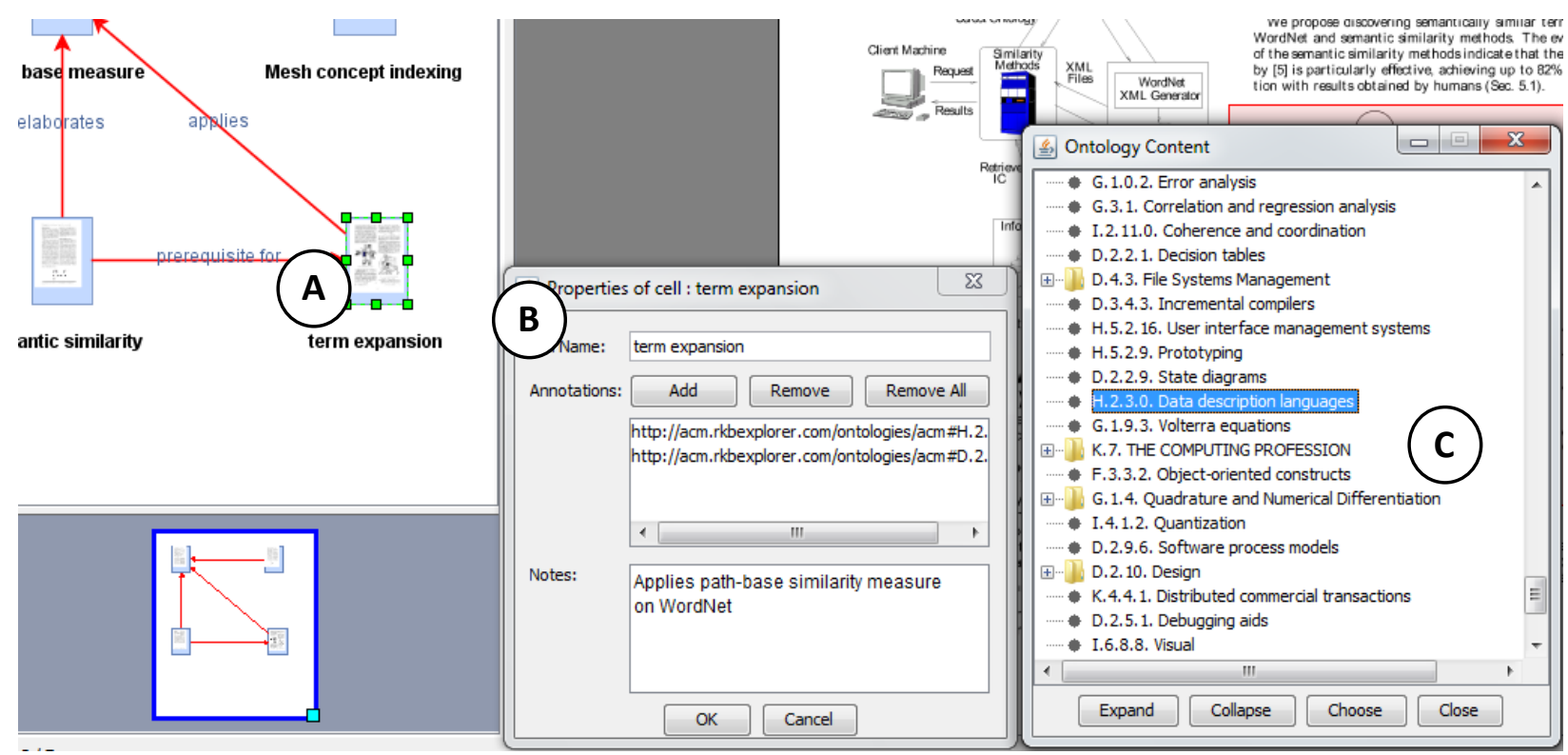

Fig 2: The annotation process in MindGate: A) the node being annotated. B) A form to input ontology-based annotations and user notes. C) A form enabling to explore the content of domain ontology and choose terms from.

Similarly, it can be difficult to understand a map constructed with MindGate without allowing users to access and read the documents referenced by the map nodes. Only after grasping the source information, users can understand the motive behind the represented structure of knowledge. Even if they read the documents, users still need to frequently refer to the map in order to recall the cognitive relationships between documents. Therefore, users will end up being distracted between the map space, which shows the knowledge structure, and the document space which shows the information content.

Our approach to resolve this issue is to merge the map space, where the knowledge map is displayed, and the document space, where the document is viewed, so that knowledge can be perceived by referring to a single space. This is done by augmenting and linking the documents in a way that matches the structure of the knowledge map. Typically, any edge connecting two nodes in the map represents a relationship between two documents. Consequently, the process is done by converting the map edges into real hyperlinks inside documents. These hyperlinks allow the user to move between the source document, denoted by the source node, and the target document, denoted by the target node, in accordance with the edge connecting them in the map.

Figure 3 illustrates in example how the conversion takes place: the paragraph enclosed by the red box (see Figure 3.C) is of interest for the user, and is thus represented by the centre node of the map (see Figure 3.A). The node has two outgoing edges named "has background" and "is explained by", and one incoming edge named as "exploits". Inside the document view, these edges are converted to a menu of hyperlinks laid over the document. Each link is named using the edge name. Clicking on a link causes the destination document to open and the target segment of interest to be highlighted. Furthermore, the attached menu includes an additional button on which pressing will show the user notes or comments (see Figure 3.D). This enables the users to view self-notes side by side with the original content. One should notice that documents are not originally linked to each other. Links between documents are dynamically created on the fly to adapt the document view to the map structure. Hence, any change made to the map structure will cause links inside documents to be changed accordingly. The transformation of graph links into physical links inside documents offers what we term "mind-map driven annotation" where documents are visually augmented and interlinked to match the cognitive structure represented as the knowledge map. We argue that the proposed adaptation process is essential for effective learning for both the knowledge creator and recipients: From the position of the knowledge provider, documents are physically linked to mimic the cognitive structure. This enables the user to focus on the navigation of the documents without having to frequently revisit the map to recall the relationships and annotations.

From the perspective of knowledge recipients, they can simply access someone's knowledge by downloading and importing its RDF file into the MindGate tool. This not only causes the knowledge map to be reconstructed on client machines, but also the documents to be interconnected using the same relationships defined in the imported map. By using the newly-attached links, a knowledge recipient can navigate documents using the same path followed by the map creator, explore how he/she cognitively linked different resources, and "live" the same experience. In addition, the names of the links explicitly reveal the creator's motivation in moving from one document to another.

\section{INFORMATION SHARING}

The MindGate tool enables users to accomplish the first step of the knowledge sharing process, which is representing knowledge graphically and adapting information content to cope with the knowledge map. The following step is to enable users to share their experience with other peers on the Web. In particular, we are interested in supporting users to publish and share their knowledge maps publically so that they can be accessed and used by other users. For this purpose, we built SocialMinds, a web based application and online repository of knowledge maps. 


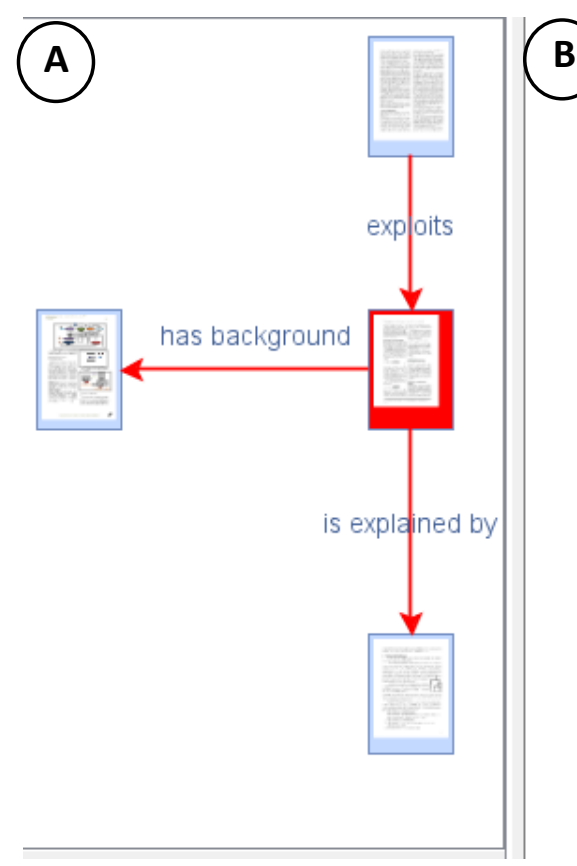

vituvgy-vascu semant measuics miv uा following three categories.

Path-based similarity Measure

Path-based similarity measure usually utilizes the information of the shortest path between two concepts, of the generality or specificity of both concepts in ontology hierarchy, and of their relationships with other concepts.

Wu and Palmer (1994) developed a similarity measure finding the most specific common concept that subsumes both of the concepts being measured. The path length from most specific shared concept is scaled by the sum of IS-A links from it to the compared two concepts. (i) has background

\begin{tabular}{lll}
$S_{W \& P}\left(\mathbf{C}\right.$ i) $\begin{array}{l}\text { is explained by } \\
\text { exploits } \\
\text { User Notes }\end{array}$ \\
\hline
\end{tabular}

In the equation (1), $N_{1}$ and $N_{2}$ is the number hierarchy, and then taking the logarithm to smooth the resulting score:

$S_{L \& C} C_{1}, C_{2} \square \square \log \square \square C_{1}, C_{2} \square 2 D$

where $\mathrm{D}$ is the maximum depth of the ontology and similarity value. In practice, we add 1 to both $d\left(C_{1}, C_{2}\right)$ and $2 D$ to avoid $\log (0)$ when the shortest path length is 0 .

Mao and Chu (2002) presented a similarity measure using both shortest path information and number of descendents of compared concepts.

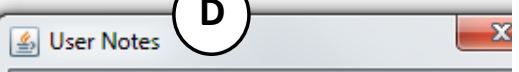

based on shortest path between concepts within the ontology

Fig 3: Mind-map driven annotation using the MindGate tool: A) The knowledge map. B) The document view. C) Map edges are converted to links inside the document. D) User notes can be displayed within the document view.

Through the MindGate tool on the client machine, a user can pack and export the constructed map as well as the referenced documents as a single file and then upload it to SocialMinds. Every user who logs in SocialMinds has a home page where he/she can access, view and publish knowledge maps. Figures 4 shows a snapshot of a user's home page where all maps uploaded by the user are listed. A snapshot of each map is created automatically and displayed to allow for rapid preview of its structure. All PDF documents referenced from the map are also listed and are available for download. SocialMinds also supports viewing and downloading maps published by other users: a map can be downloaded and reused by importing it into the MindGate tool on the client machine.

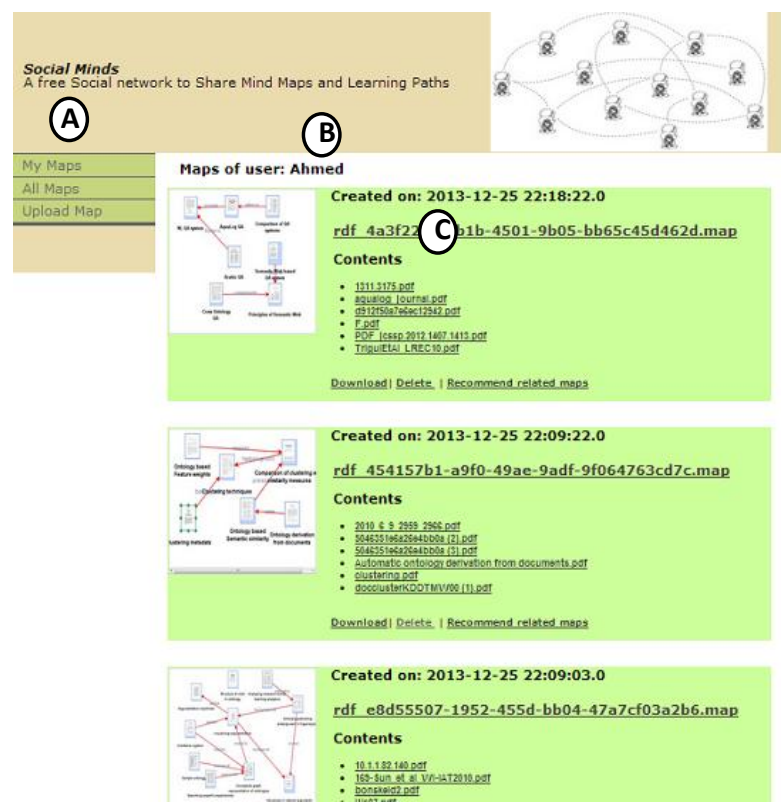

Fig 4: The main interface of SocialMinds social network showing the list of knowledge maps published by one user. A) A snapshot of each map is displayed. B) Documents referenced from the map are listed. C) Links to download or delete a map.

\section{KNOWLEDGE FLOW FROM PUBLISHER TO RECEIPENT}

This section briefly explains how the different activities and tools discussed in previous sections can be integrated together to allow for seamless transfer of knowledge. The flow of Knowledge, as depicted in Figure 5, starts by the knowledge provider who uses the MindGate tool to learn from documents and, in the meanwhile, visualize and annotate the knowledge map. Afterwards, the proposed ontology-based model is used to convert the map into a machine-understandable format that can be published and processed on the Web. SocialMinds is the web application that functions as a central repository of shared knowledge maps, and enables internet users to publish, navigate, search for and download maps.

On the other hand, knowledge recipients can download maps from the Web and reused them through the MindGate tool. This causes the entire map to be reconstructed and the documents to be interlinked in a way that reflects the relationships defined between the graph entities. Links inside documents represent the cognitive relationships made by the knowledge provider while processing information. Knowledge recipients can focus attention on the document space and use the attached links to apply experience of the knowledge provider. To conclude, our approach of knowledge sharing offers the ability to transfer the cognitive structure from the knowledge provider, in the format of an Ontologybased knowledge map, and put it at the fingertips of the knowledge recipient in the format of links and annotations inside documents.

At this stage, we revisit the criteria set by Bukowitz et al. [11] to uncover how our approach addresses the three stages for successful sharing of knowledge, which are knowledge articulation, awareness and access. Knowledge articulation is facilitated through the MindGate tool that enables users to externalize their thinking process as a map. Access to knowledge on the Web is supported by first transforming the 


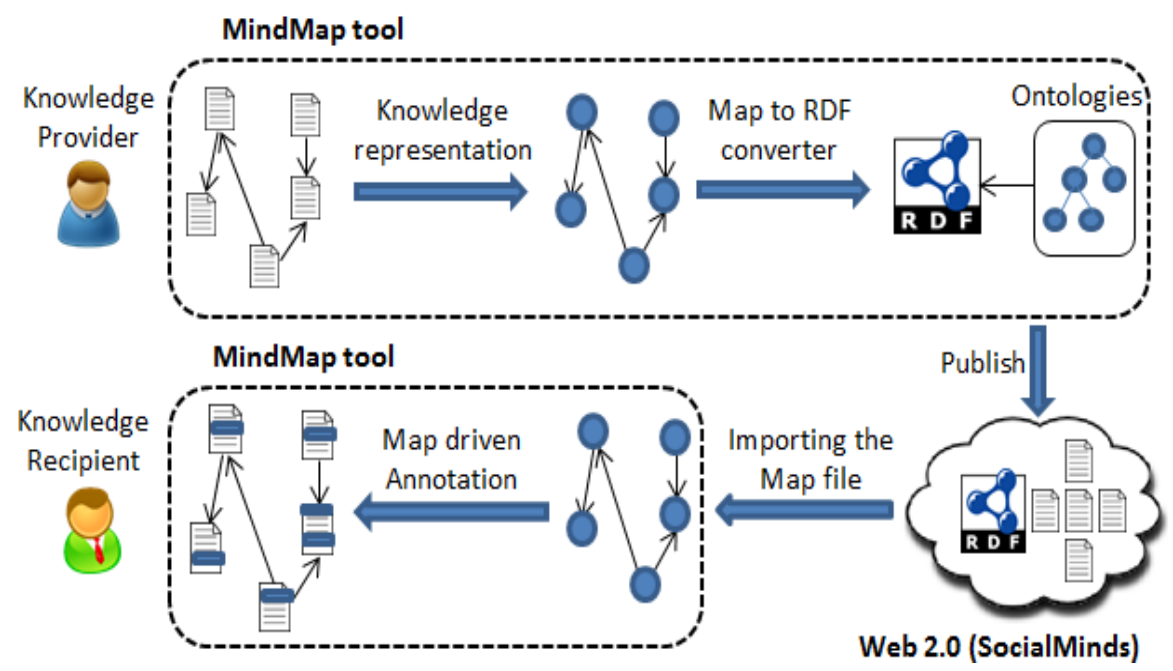

Fig 5: Knowledge flow in our approach.

knowledge map to a standardized, Web based format (e.g. RDF representation) and then providing a web based environment (e.g. SocialMinds) where maps can be shared, navigated and retrieved. Awareness of knowledge recipients can be raised by two means: First, by the "SocialMinds" application that allows users to rapidly access and retrieve maps of interest. Second, through the map-driven annotation technique that causes documents to be adapted to the structure of the knowledge map. Therefore, users can see cognitive relationships represented as links and annotations side by side with pertinent information within the document. Using the augmented view of documents, an individual can remake the learning path and recall the relationships between documents without having to be distracted by revisiting the map view frequently.

\section{USER STUDY}

In this work we proposed an integrated approach to assist users in representing, sharing and perceiving knowledge. This approach requires users to undertake a number of activities including the learning process, the construction of knowledge maps and the sharing of knowledge on the Web. Due to this variety of activities, it is difficult to assess our approach by using a single controlled experiment or by comparing it with traditional learning techniques. Thus, we opted to conduct an observational study in which a group of users were instructed to use our approach in practice. Afterwards, assessment was carried out by analyzing the use of our tools, investigating the user behaviors and exploring user preferences.

Human subjects involved in our study were three postgraduate students who were doing MSc degree in Information Technology $(2$ males and 1 females, average age $=27)$.

MSc students were individually working towards their MSc dissertations and were in the stage of writing the literature reviews on their research topics. The titles of their dissertations were as the following:

- Document clustering using multiple ontology based similarity measures.

- Design and implementation of an Arabic question answering system based on Semantic Web technologies.

- An Ontology based approach to model interconnections between research artifacts.
It is obvious that Semantic Web was the central theme of all dissertations. This was planned intentionally so that the students could have common interest to share their works.

Participants were asked to use the MindGate tool and the SocialMinds social network while doing research on their topics of study. The SocialMinds application was deployed on a local server within the University's intranet, and appropriate user accounts were created to allow participants to access and use it. Prior to the study, the MindGate and SocialMinds applications were demonstrated to the participants and they were given two hours to practice and ask questions. The duration of the study was two months. Participants were instructed to share their progress by publishing constructed maps regularly on SocialMinds.

\subsection{Data Collection}

Data was collected using the following methods:

- Applications logs: all actions made by each participant when using the MindMap tool were coded and logged in a file which was collected at the end of the study. These actions included: opening documents, manipulating knowledge graphs (creating, deleting, updating and linking graph nodes), annotating documents using domain ontology or user-notes, and saving and importing graph files. The aim of logging these details was to investigate how these features were used and whether they were useful or not.

- Similarly, all user actions performed on the SocialMinds network were logged on the server. Logged actions included the map upload and download events. These actions give an indication of the amount of knowledge maps that were shared or reused. We also maintained copies and snapshots of all knowledge maps posted by each user throughout the study.

- User Interview: participants were interviewed individually to explore their experience and perceptions.

\subsection{Analysis and Results}

We were first interested in analyzing the online activity of using the SocialMinds application. Figure 6 shows the number of actions (publish and download of maps) performed by all participants per week. It was obvious that the activity increased as the task approached its deadline. This was expected as the participants sought to share results after they were about to complete their individual work. 


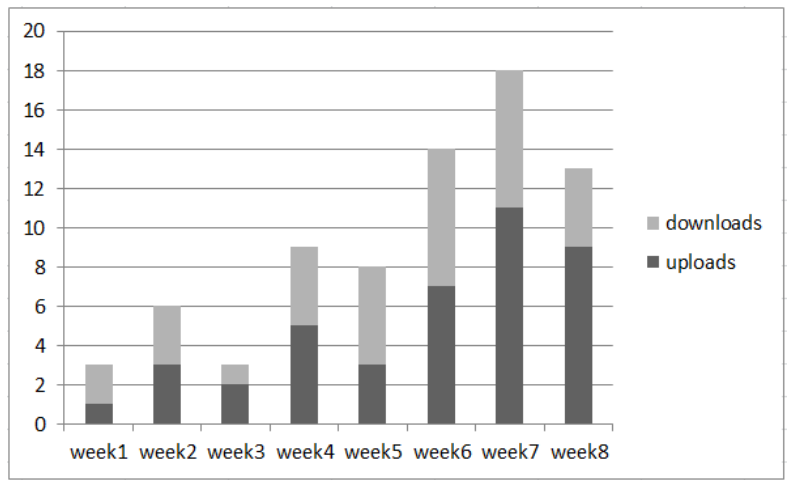

Fig 6: Average usage of the SocialMinds

The total number of publish events executed by each participant was $13.6(\mathrm{SD}=4.5)$. On tracing the snapshots of consecutive published maps, we found that participants sought to upload multiple versions of the same map whereas each version embraced updates or extensions to the former one. This could be explained by the participant's need to alter or expand the knowledge map progressively during the task. Besides, each participant executed eleven download actions on average $(\mathrm{SD}=3)$ to access maps shared by other participants. This indicates that participants used the SocialMinds continually to share their work as well as to access others' work. To give a remark on how well the participants managed to represent knowledge visually, the maps uploaded by participants were inspected. On average, each participant referenced eleven documents $(\mathrm{SD}=5.2)$ from within the map, and used them to make 15 links on average $(\mathrm{SD}=6.1$ ) between the contents of these documents.

Looking at the links between graph nodes, it was obvious that participants often managed to name links between nodes properly to reflect the desired relationship between the corresponding documents. For example, Figure 7 shows a sample map published by one participant. Looking at the map edges, we can find names such as "applies", "requires", "related to". These names show that participants could seamlessly visualize the relationships between information items and name them appropriately. Besides the analysis of user activity on the SocialMind's application, we were interested in exploring how participants used the MindGate tool individually while building their knowledge maps.

The analysis of the application log for the SocialMinds tool revealed that participants built their maps over several weeks. Participants needed multiple sessions to complete the task. Each participant needed an average of 6.2 sessions $(S . D=3.5)$ that were spread throughout the task duration ( 8 weeks). A single session was determined by the start and end times that were logged once the map file was opened and closed respectively. Of the actions we analyzed, a remarkable observation was the considerable number of revisits to the documents: At the beginning of each session, each participant often needed to revisit documents from previous sessions to review knowledge and recall what was learned before. We noticed that participants used the layer of links embedded inside documents intensively to make the revisits. This was indicated by the number of click events made on these links. This result reveals an additional benefit of our approach in that it is not only useful for knowledge sharing and publishing, but is also useful for individual work by supporting self-revision. Individuals can rapidly navigate the documents in accordance with the graph constructed on the drawing panel using the newly-created links.
When interviewed, participants were first asked about their impression about the overall activity and whether the presented tools helped them complete the given task. They all gave positive feedback: One participant said that he used to have many difficulties when trying to retain links between existing research documents. With the MindGate tool, he can better organize work and link references in a network-like structure while enabling easy navigation from one reference to another. All Participants indicated that they can easily download, import and use the work of others. One participant indicated that the newly-attached links and annotations were extremely useful especially when the knowledge map got

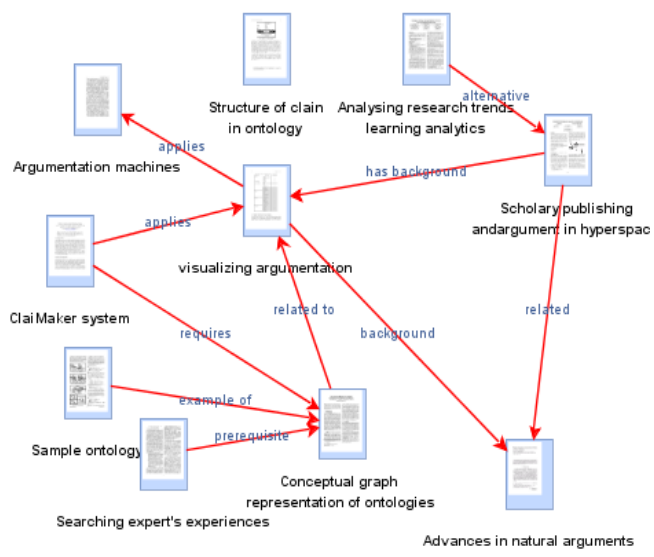

Fig 7: A sample knowledge map

bigger and became difficult to trace.

When asked about the usefulness of the maps shared on the SocialMinds application, two participants mentioned that the shared maps helped them discover some important research efforts that they were not aware of, as well as gain a thorough understanding of the research background. One participants liked the ability to import someone's work and follow the same learning path as he/she did through the links created inside documents.

Participants also reported some limitations and ideas to enhance our work: one participant mentioned that the ability to make links between graph nodes is not enough to fully visualize everything in mind: While a graph node represents a document, or a segment of a document, a user sometimes needs to represent different entities such as topics, concepts or ideas and make links between them. A user may also need to classify some documents by putting them into different groups. This triggers the need to extend the MindGate so that more flexibility and expressiveness can be achieved in the knowledge representation stage.

\section{CONCLUSION AND FUTURE WORK}

In this work, we presented an integrated approach for knowledge sharing that addresses the needs of both knowledge providers and recipients. Our approach offers a solution to facilitate the activities of knowledge representation, transfer and access. When compared with previous efforts, our work makes the following contributions: First, our approach of knowledge sharing is not limited to the prevailing paradigm of viewing and sharing information online. Rather, it goes a step forward by offering "pluggable knowledge components" in which the documents, the cognitive relationships and the user-define annotations can all be communicated, reconstructed and reused on any machine. Second, we offer a content-adaptation technique that we termed "knowledge map driven annotation". In this technique, 
the knowledge graph constructed by the user is automatically converted to a layer of links and annotations over the source documents. As a consequence, users can view documents and, at the same time, can still understand how the content has been structured and interlinked to make the entire knowledge.

Our approach has been preliminarily evaluated by a group of researchers within the context of a realistic learning task. The analysis of the user behavior and the interaction with applications revealed the potential of our approach to facilitate knowledge articulation, transfer and perception.

In our future work, we will address the limitations and ideas raised by the participants. We will also explore how our approach can generalize to various learning scenarios by testing it with a larger number of participants and different domains of knowledge.

\section{REFERENCES}

[1] Argote, L. and P. Ingram, Knowledge transfer: A basis for competitive advantage in firms. Organizational behavior and human decision processes, 2000. 82(1): p. 150-169.

[2] Wiig, K.M., What future knowledge management users may expect. Journal of knowledge management, 1999. 3(2): p. $155-166$

[3] Szulanski, G., The process of knowledge transfer: A diachronic analysis of stickiness. Organizational behavior and human decision processes, 2000. 82(1): p. 9-27.

[4] Kogut, B. and U. Zander, Knowledge of the firm, combinative capabilities, and the replication of technology. Organization science, 1992. 3(3): p. 383397.

[5] Paroutis, S. and A. Al Saleh, Determinants of knowledge sharing using Web 2.0 technologies. Journal of knowledge management, 2009. 13(4): p. 52-63.

[6] Chi, E.H., The Social Web: Research and Opportunities. IEEE Computer, 2008. 41(9): p. 88-91.

[7] Hollan, J., E. Hutchins, and D. Kirsh, Distributed cognition: toward a new foundation for human-computer interaction research. ACM Transactions on ComputerHuman Interaction (TOCHI), 2000. 7(2): p. 174-196.

[8] Hendriks, P., Why share knowledge? The influence of ICT on the motivation for knowledge sharing. Knowledge and process management, 1999. 6(2): p. 91100 .

[9] Hew, K.F., Knowledge sharing among professionals in three online communities. 2006: ProQuest.

[10] Tuomi, I. Data is more than knowledge: implications of the reversed knowledge hierarchy for knowledge management and organizational memory. in System Sciences, 1999. HICSS-32. Proceedings of the 32nd Annual Hawaii International Conference on. 1999: IEEE.

[11] Bukowitz, W.R., R.L. Williams, and F. Times, The knowledge management fieldbook. 1999: Financial Times Prentice Hall.

[12] Sowa, J.F., Knowledge representation: logical, philosophical, and computational foundations. Vol. 13. 2000: MIT Press.
[13] Berners-Lee, T., J. Hendler, and O. Lassila, The semantic web. Scientific american, 2001. 284(5): p. 28-37.

[14] Buzan, T. and B. Buzan, The mind map book. 2000: BBC.

[15] Eppler, M.J., Making knowledge visible through knowledge maps: concepts, elements, cases. Handbook on knowledge management, 2003. 1: p. 189-205.

[16] Adodo, S., Effect of Mind-Mapping as a Self-Regulated Learning Strategy on Students Achievement in Basic Science and Technology. Mediterranean Journal of Social Sciences, 2013. 4(6): p. 163.

[17] Eppler, M.J., A comparison between concept maps, mind maps, conceptual diagrams, and visual metaphors as complementary tools for knowledge construction and sharing. Information Visualization, 2006. 5(3): p. 202210 .

[18] Huff, A.S. and M. Jenkins, Mapping strategic knowledge. 2002: SAGE Publications Limited.

[19] Coyne, R., Designing information technology in the postmodern age: From method to metaphor. 1995: Mit Press.

[20] Gouli, E., et al. COMPASS: an adaptive web-based concept map assessment tool. in Proceedings of the first international conference on concept mapping. 2004.

[21] Novak, J.D. and A.J. Cannas, The origins of the concept mapping tool and the continuing evolution of the tool. Information Visualization, 2006. 5(3): p. 175-184

[22] AlAgha, I., KnowledgePuzzle: A Browsing Tool to Adapt the Web Navigation Process to the Learner's Mental Model. Educational Technology \& Society, 2012. 15(3): p. 275-287.

[23] Cannas, A.J., et al. CmapTools: A knowledge modeling and sharing environment. in Concept maps: Theory, methodology, technology. Proceedings of the first international conference on concept mapping. 2004.

[24] Selvin, A., et al., Compendium: Making meetings into knowledge events. 2001

[25] Hwang, G.-J., Y.-R. Shi, and H.-C. Chu, A concept map approach to developing collaborative Mindtools for context-aware ubiquitous learning. British Journal of Educational Technology, 2011. 42(5): p. 778-789.

[26] Mancini, C. and S.J. Buckingham Shum, Modelling discourse in contested domains: A semiotic and cognitive framework. International Journal of Human-Computer Studies, 2006. 64(11): p. 1154-1171.

[27] Uren, V., et al., Semantic annotation for knowledge management: Requirements and a survey of the state of the art. Web Semantics: science, services and agents on the World Wide Web, 2006. 4(1): p. 14-28.

[28] Hillmann, D., Using Dublin Core, 2001. URL http://dublincore. org/documents/2001/04/12/usageguide/.

DCMI Recommendation, 2001

[29] Hwang, W.-Y. and G.-L. Hsu, The effects of pre-reading and sharing mechanisms on learning with the use of annotations. TOJET, 2011. 10(2). 
[30] Marshall, C.C. and A.B. Brush. Exploring the relationship between personal and public annotations. in Digital Libraries, 2004. Proceedings of the 2004 Joint ACM/IEEE Conference on. 2004: IEEE.

[31] Su, A., et al., A Web 2.0-based collaborative annotation system for enhancing knowledge sharing in collaborative learning environments. Computers \& Education, 2010. 55(2): p. $752-766$.

[32] Cress, U., C. Held, and J. Kimmerle, The collective knowledge of social tags: Direct and indirect influences on navigation, learning, and information processing. Computers \& Education, 2012.

[33] Oren, E., et al., What are semantic annotations. Relatório técnico. DERI Galway, 2006.
[34] Jung, J.J., Consensus-based evaluation framework for distributed information retrieval systems. Knowledge and Information Systems, 2009. 18(2): p. 199-211.

[35] Wang, Z., Q. Wang, and D.-W. Wang, Bayesian network based business information retrieval model. Knowledge and Information Systems, 2009. 20(1): p. 63-79.

[36] Wang, P., et al., Using Wikipedia knowledge to improve text classification. Knowledge and Information Systems, 2009. 19(3): p. 265-281.

[37] Wong, T.-L. and W. Lam, Learning to extract and summarize hot item features from multiple auction web sites. Knowledge and Information Systems, 2008. 14(2): p. $143-160$. 